\title{
Universiteit
}

Leiden

The Netherlands

\section{Quantum critical electron systems: the uncharted sign worlds}

Zaanen, J.

\section{Citation}

Zaanen, J. (2008). Quantum critical electron systems: the uncharted sign worlds. Science, 319(5867), 1205-1207. doi:10.1126/science.1152443

\author{
Version: $\quad$ Publisher's Version \\ License: $\quad$ Licensed under Article 25fa Copyright Act/Law (Amendment Taverne) \\ Downloaded from: $\quad$ https://hdl.handle.net/1887/3158104
}

Note: To cite this publication please use the final published version (if applicable). 
particle state and thus to behave in exactly the same way [compare with (6)]. This property leads to a variety of spectacular effects, including the complex of phenomena known as superfluidity, which is observed to occur in the He-II phase of liquid ${ }^{4} \mathrm{He}$, and a variety of interference phenomena, which it has become possible to observe in the ultracold atomic gases [(2), section 2.5].

Turning now to Fermi systems, we see from Eq. 2 that in this case the value of $\left\langle n_{k \sigma}\right\rangle$ can never exceed 1 (the Pauli principle), so that the direct analog of BEC certainly cannot occur. However, there is no reason why a complex made up of an even number of fermions (a boson) cannot undergo BEC (indeed, this is exactly what is happening in, for example, ${ }^{87} \mathrm{Rb}$ at ultralow temperatures), and in particular there is every reason to believe (compare below) that if two spin-1/2 fermions are coupled by an attractive interaction sufficiently strong to bind them into a spin- 0 bosonic molecule, these molecules will indeed undergo BEC; such a scenario might be imagined to describe, for example, liquid $\mathrm{D}_{2}$, if we could exclude crystallization. Imagine now that we gradually weaken the interfermion attraction to the point where (in the two-body problem) the molecule is no longer stable and even beyond. Can a sort of BEC still persist under these conditions? In their epochmaking work (7) in 1957, Bardeen, Cooper, and Schrieffer (BCS) showed that the answer is yes: A degenerate system of fermions with an arbitrarily weak attraction will, at sufficiently low temperatures (exponentially low compared to $T_{\mathrm{d}}$ ), form "Cooper pairs," a sort of giant di- atomic (or more accurately dielectronic, because BCS were dealing explicitly with the electrons in a metal), spin-0 molecules, and the latter will then in effect automatically undergo the phenomenon of BEC. In contrast to the case of (hypothetical) liquid $\mathrm{D}_{2}$, however, the size of the "molecules" is now large compared with their average separation, so that the theory of Cooper pairing is quantitatively and even qualitatively quite different from that of BEC of tightly bound diatomic molecules.

When Cooper pairing occurs in an electrically neutral system of fermions such as liquid ${ }^{3} \mathrm{He}$, the consequences are qualitatively similar to those of BEC in a bosonic system such as ${ }^{4} \mathrm{He}$. Indeed, it is almost universally believed that the anomalous phases of liquid ${ }^{3} \mathrm{He}$ that occur below $3 \mathrm{mK}$ (note this is $<<T_{\mathrm{d}} \sim 1 \mathrm{~K}$ ), which show many of the manifestations of superfluidity, are indeed characterized by the onset of Cooper pairing. When the latter occurs in the electrically charged system of electrons in metals, the effects are even more spectacular: In particular, the metal in question will exhibit the two major effects characterizing superconductivity, namely, persistent flow of currents in a ring and the exclusion of magnetic flux (Meissner effect), which leads to the possibility, inter alia, of static magnetic levitation. Superconductivity, originally thought to be an intrinsically low-temperature effect, has in the past 20 years been observed to occur in a class of cuprate materials up to around half of room temperature; although the detailed explanation of this high-temperature superconductivity is still furiously debated, there seems little doubt that its fundamental origin lies in the phenomenon of Cooper pairing.

As explained above, it is somewhat natural to think of the phenomenon of Cooper pairing in a system of fermions with weak attraction on the one hand and BEC in the system of diatomic molecules formed from them on the other as opposite ends of the same spectrum, and it has long been speculated that by "tuning" the strength of the attraction one might be able to realize a continuous transition between the two situations; this is known as the "BEC-BCS crossover." In the past 4 years, by using the phenomenon of Feshbach resonance, it has become possible to study the BEC-BCS crossover experimentally in ultracold atomic gases, and it indeed appears to be continuous as tentatively predicted by theory: See the article by I. Bloch in this issue (3). Thus, we now have a very satisfying unification of the concepts of BEC in a bosonic system and Cooper pairing in a fermionic one.

References and Notes

1. R. F. Streater, A. S. Wightman, PCT, Spin and Statistics and All That (Benjamin, New York, 1964).

2. A. J. Leggett, Quantum Liquids: Bose Condensation and Cooper Pairing in Condensed-Matter Systems (Oxford Univ. Press, Oxford, 2006).

3. I. Bloch, Science 319, 1202 (2008)

4. A. Einstein, Abh. Preuss. Akad. Wiss. 3, 18 (1925).

5. M. R. Andrews et al., Science 273, 84 (1996).

6. Molecule of the Year for 1995, Science 270 (1995).

7. J. Bardeen, L. N. Cooper, J. R. Schrieffer, Phys. Rev. 108, 1175 (1957)

8. This work was supported by the NSF under grant no. NSFDMR-03-50842.

10.1126/science.1152822

\section{Quantum Critical Electron Systems: The Uncharted Sign Worlds}

\section{J. Zaanen}

Phases of classical matter, such as solids and liquids, are ruled by emergence principles that are well understood. Although the same principles govern forms of quantum matter that have no secrets for physicists, such as the superfluids, having to deal with fermions and the associated Fermi sign problem shatters this analogy. This Perspective addresses the Fermion sign problem and describes experiments on metals undergoing quantum phase transitions exhibiting scale-invariant electronic behavior, a description of which is at odds with established quantum theory.

$\mathrm{I}$ ce is different from water, and water is different from steam, although these phases of matter are all made from the same water molecules. Countless numbers of molecules are required to make this work, and the various phases of matter are said to "emerge." Emergence is at its best

Instituut-Lorentz for Theoretical Physics, Leiden University, Leiden 2333 CA, Netherlands. E-mail: jan@lorentz. leidenuniv.nl

when the transition between such phases is continuous and the system no longer has any sense of preference for one or the other phase. This lack of "executive power" has the consequence that the system spontaneously adopts the powerful symmetry of scale invariance. In this "critical state" the system looks on average the same, regardless of the amplification factor that is used to observe it (1).

The concept of emergence is so powerful that it transcends the classical-quantum divide, and this apparently includes the generation of scale invariance: Quantum critical states where quantum fluctuations drive a phase transition at zero temperature are now routinely observed (2-6). However, dealing with emergence in quantum physics requires one to consider the organizational principles of quantum statistics, as discussed by Leggett (7), whereby quantum particles are either bosons or fermions. Despite its underlying quantum properties, bosonic matter is ruled by the same emergence principles as classical matter (2). In stark contrast, Fermi statistics wreck this analogy, and the emergence principles governing fermionic matter are among the great mysteries of modern physics. Fortunately, experimentation can help: Electrons in solids are relatively easy to probe, and they form systems of countless numbers of strongly interacting fermions. The recently observed quantum phase transitions in a variety of metals (3-6) reveal that fermionic quantum matter can exhibit unexpected behavior: Particles tend to acquire an infinite mass, and the scale-invariant fermionic phases that take over appear to be the birthplace of new forms of stable quantum matter.

Regular matter is formed from a large number of quantum particles, electrons, quarks, and 
so forth. To answer the question of where classical integrity comes from, we look to Feynman's path integral formalism (2), a rather pictorial view of the quantum world but one that does reproduce accurately all the known facts about quantum systems in equilibrium. Within this formalism, quantum systems resemble classical matter living in a higherdimensional "Euclidean spacetime" (Fig. 1) having an extra axis, "imaginary time" (our time times the square root of -1$)$. The maximal duration of this imaginary time is the ratio of Planck's constant divided by temperature: When temperature is lowered, "more time is available" to see quantum behavior. The strength of the quantum fluctuations is analogous to temperature in classical physics, having the effect of "heating up" the "stuff" inside space-time. When temperature is lowered and the quantum fluctuations are sufficiently vigorous, this frozen quantum matter might melt. The resulting space-time liquid will appear to our eyes as, for instance, a superconductor. When this melting transition taking place in space-time at zero temperature generates scale invariance, the quantum critical state is realized.

These quantum critical states have in fact become quite ubiquitous in the laboratory. One variety is formed by the "designer quantum critical states," where the theorists have so much understanding that they can now guide the experimentalists to where to look. Prominent examples are the cold atoms and the spin systems, as highlighted by Bloch ( 8 ) and Lloyd (9). For bosons and some spin systems, the stuff filling up the space-time of the path integral is similar to classical matter, but this is not at all the case for fermions. The culprit for this deviation is the infamous "fermion sign problem." When fermions come into play, it turns out that in the statistics underlying the description of matter, one must deal with "negative probabilities," and this detaches the many-fermion problem from any classical analog. We have in fact no understanding at all of what is going on in space-time, because we need mathematics to look around and the sign problem is "NP hard" (10), meaning that the problem is mathe-

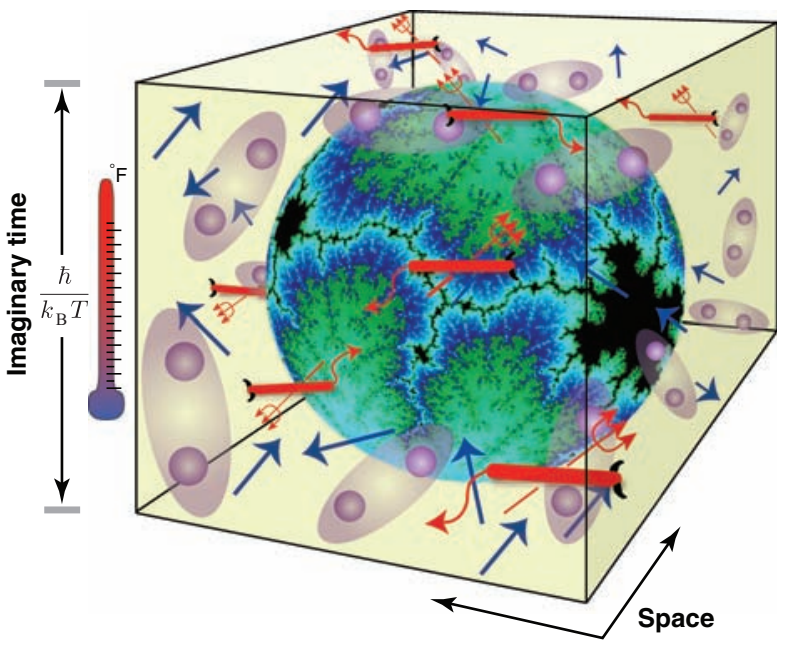

Fig. 1. Illustrating the Feynman path integral, the mathematical tool of choice to address emergence phenomena in many-particle quantum systems (2). Near a quantum phase transition, the world inside spacetime turns scale-variant at shorter scales, like the Julia set of this cartoon, whereas at larger scales a stable form of quantum matter takes over. Dealing with fermions, the devilish minus signs obscure, however, any detailed understanding of these space-time worlds. The duration of imaginary time is determined by $\hbar$ (Planck's constant divided by $2 \pi$ ) and the product of Boltzmann's constant $k_{\mathrm{B}}$ and absolute temperature $T$.

matically unsolvable. The only fermionic substance that we can handle mathematically is the Fermi liquid, the state of electrons in normal metals. Although different from any form of classical matter, this state is at first sight deceptively simple: The electrons turn cooperatively into noninteracting "quasi-electrons" that only communicate

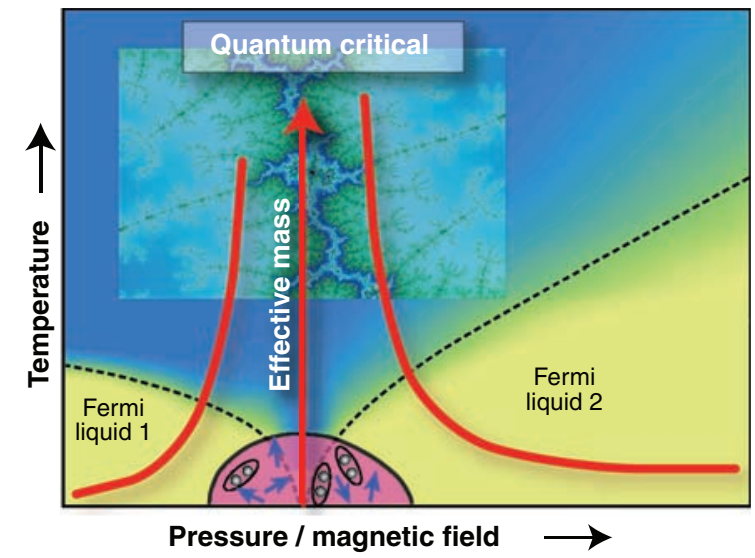

Fig. 2. Typical phase diagram observed in the heavy-fermion metals in the proximity of a quantum phase transition (3-6). The thermal phase transition to a magnetic state is driven to zero temperature by varying a magnetic field or pressure, and this is the anchor point of a regime of finite-temperature quantum critical fluid behavior fanning out for increasing temperature. The fermionic weirdness manifests itself through the effective mass of the quasi-electrons in the Fermi liquids on both sides, which increases without bound approaching the quantum phase transition. Invariably one finds that at a low temperature, an exotic superconductor (or even a quantum liquid crystal state) takes over at the last minute. via the Pauli exclusion principle. This pushes them into states of high quantum zero-point energy (7), and their average "Fermi energy" is typically on the order of $10,000 \mathrm{~K}$ in standard metals. This Fermi liquid is in fact a purely empirical construction. Although it is observed in experiments, theoretical physics has failed to explain its existence in general terms, despite countless attempts.

But electrons are also known to form "nonFermi liquid" states, as can be found in metals containing rare earth, actinide, and transition metal ions (3-6). At ambient conditions, these show a phase transition to some magnetic state at a low temperature, and by applying pressure or magnetic fields, this transition can be driven to zero temperature. For example, in the diagram of pressure/ magnetic field versus temperature (Fig. 2), one finds a V-shaped region anchored where the magnetic transition approaches zero temperature, and the regime inside the $\mathrm{V}$ shows the telltale signs of the quantum critical fluid. Actually, this fanning out of the quantum critical region for increasing temperature is just mapping out the "scale invariance geography" in space-time. When the system is close but not at the phase transition, it will show the physics of the stable phase at large scales (Fig. 1). However, upon zooming in, the system will forget its preferred state, and at a characteristic scale the system will reenter the scale-invariant regime. The increase of temperature is like the magnification factor of a microscope, and the $\mathrm{V}$ reveals that the scale where the system takes the decision to become a stable phase shifts to shorter times when one moves away from the quantum critical point.

But now the fermion signs hit hard: The experiments give away the workings of quantum scale invariance in space-time, but we have no clue whatever about the nature of the stuff creating the scale invariance! The stable states that are found outside the $\mathrm{V}$ in the proximity of the quantum phase transition are Fermi liquids, and because we have a phenomenological understanding of these states, they tell us something. When interactions are weak, one can do controlled calculations, and these reveal a peculiar Fermi liquid rule: The interactions between real electrons have the effect of increasing the mass of the quasi-electrons. This mass enhancement effect is quite modest when the calculations can be trusted, but in the approach to the metallic quantum phase transition, one finds that the effective mass of these quasi-electrons easily exceeds 1000 times the electron mass, to increase indefinitely upon getting closer and closer to the quantum critical point $(3,4)$. Again the only hold we have is quantum scale invariance: The Fermi energy is a scale, but because the quantum critical state forbids any scale, it has to disappear. The Fermi energy is the average zero-point motion energy, and the only way to remove it is by making the mass of the quasi-electrons infinite! 
Somehow there is something badly wrong with these infinitely heavy quasi-electrons. Nature seems to share this concern: Without exception, one observes that eventually some other stable quantum matter state takes over (Fig. 2). These phenomena are currently under intense investigation, and it is clear that they can be quite strange. Recently a quantum version of a liquid crystal was discovered (5) but generically strange forms of superconductivity were found $(3,4)$, including a superconductor that appears to be indestructible by magnetic fields (6).

These observations beg for an explanation in terms of a triumphant mathematical theory, but the efforts of the theorists have gotten stuck in running variations on the established themes of bosonic matter and the Fermi liquid (4): One finds the fermion signs, in one or the other disguise, as the proverbial brick wall blocking any progress. The "heavy fermion" quantum criticality highlighted here is quite instrumental in forcing us to face the fact that there is still a vast quantum territory lying behind our intellectual horizon that awaits further exploration. The 20-year-long struggle of the physics community with superconductivity at high temperatures, as found in copper oxides, might well be rooted in the sign problem: Although the empirical situation is less clear, there are indications that this high- $T_{\mathrm{c}}$ superconductivity is born from a quantum critical state (11). But the fermion signs infest all of physics. In high-energy physics this is well recognized in the context of quark matter, but it might even be consequential in the most fundamental realms (12). The modern way of thinking about the ultimate origin of space-time (and everything else) has quantum emergence as a common denominator, but even string theory rests in this regard on intuitions originating in the earthly realms. There are plenty of fermions in such theories, but they are instinctively taken to be of the Fermi-liquid kind, and there is plenty of room for big surprises caused by the fermion signs at the very bottom.

\section{References and Notes}

1. ]. Cardy, Scaling and Renormalization in Statistical Physics (Cambridge Univ. Press, Cambridge, 1996).

2. S. Sachdev, Quantum Phase Transitions (Cambridge Univ. Press, New York, 1999).

3. P. Coleman, A. J. Schofield, Nature 433, 226 (2005).

4. H. von Löhneisen, A. Rosch, M. Vojta, P. Wölfle, Rev. Mod. Phys. 79, 1015 (2007).

5. R. A. Borzi et al., Science 315, 214 (2007); published online 22 November 2006 (10.1126/science.1134796).

6. F. Levy, I. Sheikin, A. Huxley, Nature Phys. 3, 460 (2007).

7. A. J. Leggett, Science 319, 1203 (2008)

8. I. Bloch, Science 319, 1202 (2008).

9. S. Lloyd, Science 319, 1209 (2008).

10. M. Troyer, U. J. Wiese, Phys. Rev. Lett. 94, 170201 (2005). 11. J. Zaanen, Nature 430, 513 (2004) and references therein.

12. H. Georgi, Phys. Rev. Lett. 98, 221601 (2007).

13. I thank F. Krueger for his work on the figures.

$10.1126 /$ science. 1152443

\section{PERSPECTIVE}

\section{Supersolidity}

\section{H. W. Chan}

The observation of nonclassical rotational inertia (NCRI) by the torsional oscillator in 2004 gave rise to a renaissance in the study of solid helium-4. Recent theoretical and experimental studies found evidence that disorder in the solid plays a key role in enabling superfluidity. A recent experiment found a marked increase in the shear modulus that shares the same temperature and helium-3 impurity concentration dependence as that of NCRI. This correlation indicates that the onset of superfluidity requires the pinning and stiffening of the dislocation network by helium-3.

$\mathrm{S}$ hortly after the discovery of superfluidity in liquid ${ }^{4} \mathrm{He}(1,2)$, the possibility of the same phenomenon occurring in solid helium was raised by Wolfke (3). Careful theoretical consideration of the problem (4-7) suggested that the possible presence of quantum mechanically induced or zero-point lattice vacancies could facilitate such a "supersolid." In this scenario the superfluid fraction, which reaches $100 \%$ in liquid helium, may be immeasurably small. Nevertheless, the suggestion spurred considerable experimental effort in search of evidence for the supersolid phase. Other than some interesting anomalies in the ultrasound experiments $(8)$, these efforts were unsuccessful (9). The situation changed in 2004 when we reported $(10,11)$ superfluid-like behavior of solid helium samples housed within a torsional oscillator (TO).

In an ideal TO the resonant period is given by $2 \pi(I / G)^{1 / 2}$, where $G$ is the torsional spring constant of the torsion rod and $I$ the rotational inertia of the torsion bob. We observed that below $200 \mathrm{mK}$, the resonant period of such an oscillator

Department of Physics, Pennsylvania State University, University Park, PA 16802, USA. E-mail: chan@phys. psu.edu iments led us to conclude that the period drop was due to the solid ${ }^{4} \mathrm{He}$ confined inside the torsion bob oscillating with an effective moment of inertia $I$ that is smaller than the high-temperature, classical value. This is known as nonclassical rotational inertia (NCRI), where $I(T)=I_{\text {classical }}[1-$ $\left.f_{\mathrm{s}}(T)\right]$ and $f_{\mathrm{s}}(T)$ is the superfluid fraction (7). $f_{\mathrm{s}}$ becomes distinguishable from noise at an onset temperature, $T_{0} \sim 200 \mathrm{mK}$, and grows at first gradually and then more rapidly with decreasing temperature before saturating below $\sim 50 \mathrm{mK}$ (Fig. 1). We found $f_{\mathrm{s}} \sim 1 \%$ in the low-temperature limit for solid samples grown inside an annulus of $1 \mathrm{~mm}$ in width, as well as for those confined within porous structures having characteristic lengths from nanometers (10) to half a micrometer (12). The measured value of $f_{\mathrm{s}}$ is attenuated when the oscillation speed exceeds a value corresponding to several quanta of circulation, suggesting that the important excitations in the system are vortices. The phenomenon is immensely sensitive to ${ }^{3} \mathrm{He}$ impurities, even down to a concentration of $x_{3}$ in the 1 part per billion (ppb) level. The temperature at which $f_{\mathrm{s}}$ reaches half its saturated value, $T_{1 / 2}$, increases smoothly from $30 \mathrm{mK}$ at $x_{3}=1 \mathrm{ppb}$ to $500 \mathrm{mK}$ at $x_{3}=85$ parts per million (ppm) $(10,13)$. drops rather abruptly. A number of control exper-
A phenomenological model that captures a great deal of the experimental findings is the vortex liquid model proposed by Anderson (14). The attenuation of $f_{\mathrm{s}}$ with oscillation speed is attributed to the nonlinear susceptibility of the entangled collection of many thermally activated vortices. The ability of the vortices to move counter to the time-dependent superflow (relative to the cell's oscillation) results in the screening of the supercurrents. As the temperature is lowered, the motion and number of vortices are reduced so that $f_{\mathrm{s}}$ becomes finite. One prediction of the model is an increase in $T_{0}$ with increasing measurement frequency, and it was confirmed for the same sample $\left(x_{3}=\right.$ $0.3 \mathrm{ppm})$ that $T_{0} \sim 160 \mathrm{mK}$ at $496 \mathrm{~Hz}$ and $T_{0} \sim$ $240 \mathrm{mK}$ at $1173 \mathrm{~Hz}(15)$.

The observation of NCRI has now been replicated in at least three other laboratories (16-19). Although the temperature dependence of $f_{\mathrm{s}}(T)$ is entirely reproducible, its magnitude varies substantially. The low-temperature supersolid fraction ranges from as little as $0.015 \%$ to as much as $20 \%$, the latter of which was reported by Rittner and Reppy in their studies of extremely narrow annuli $(0.15 \mathrm{~mm}$ width) of solid helium (17). They also found $f_{\mathrm{s}}$ to be substantially reduced by thermally annealing the sample (16). The large variation in $f_{\mathrm{s}}$, the effects of annealing, and the lack of evidence for zero-point vacancies (20) in the $T=0$ limit support the theoretical consensus that superfluidity does not exist in a perfect crystal $(20,21)$.

Three types of disorder have been considered to be responsible for the phenomenon: glassy regions, grain boundaries, and dislocation lines. Glassy regions have been proposed primarily because they lack crystalline order, thus making them more amenable for superfluidity. Indeed, a quantum Monte Carlo simulation (22) found that when disorder is quenched into the 


\title{
Science
}

\section{Quantum Critical Electron Systems: The Uncharted Sign Worlds}

\author{
J. Zaanen
}

Science 319 (5867), 1205-1207.

DOI: $10.1126 /$ science. 1152443

ARTICLE TOOLS

RELATED
CONTENT

REFERENCES

PERMISSIONS http://science.sciencemag.org/content/319/5867/1205

http://science.sciencemag.org/content/sci/319/5867/1201.full

This article cites 10 articles, 4 of which you can access for free http://science.sciencemag.org/content/319/5867/1205\#BIBL

http://www.sciencemag.org/help/reprints-and-permissions 